\title{
Caracterização Morfológica de Nanomembranas de Poliamida-66 Dopadas com Grafeno Obtidas por Electrospinning
}

\author{
José de Ávila Júnior \\ Programa de Pós-graduação em Engenharia Mecânica, UFMG \\ Antônio Ferreira Ávila \\ Departamento de Engenharia Mecânica, UFMG
}

\author{
Matt H. Triplett \\ Aviation and Missile Research Development and Engineering Center, AMSRD-AMR-WD-GA
}

\begin{abstract}
Resumo: Neste estudo, investigou-se a síntese de nanomembranas por eletrofiação (electrospinning). A poliamida-66 (PA-66) foi usada para estudar a influência dos parâmetros operacionais (concentração do polímero, diferença de potencial elétrico aplicada, vazão da solução, distância entre ponta da agulha e o coletor) na morfologia das nanofibras. Também foi estudado o efeito da adição de nanofolhas de grafeno na morfologia das nanofibras. Os resultados demonstraram que o diâmetro médio das nanofibras é diretamente proporcional à vazão, à concentração do polímero e à diferença de potencial elétrico aplicada. A adição de nanopartículas de base carbono fez com que o diâmetro médio das nanofibras aumentasse. Os diâmetros médios para as concentrações de grafeno de $0 \%$, $1 \%$, e $2 \%$ variaram de $57 \mathrm{~nm}(0 \%)$ até $141 \mathrm{~nm}(2 \%)$. No entanto, é importante salientar que os diâmetros médios das nanofibras obtidas estão $37 \%$ menores que aqueles reportados na literatura.
\end{abstract}

Palavras-chave: Electrospinning, nanofibras, nanomembranas, grafeno, compositos nano-estruturados.

\section{Morphological Characterization of Polyamide-66 Nanomembranes with Graphene Obtained by Electrospinning}

\begin{abstract}
This paper reports on the synthesis of polymeric nanomembranes produced by electrospinning. Polyamide-66 (PA-66) was used for studying the influence of fabrication parameters (polymer concentration, applied tension, solution flow rate, gap between needle and target) on the morphology of the nanofibers. Also investigated was the effect from adding graphene into the nanofiber. The average diameter was directly proportional to polymeric concentration, flow rate, and applied tension. The addition of graphene led to an increase in the average diameter, which ranged from $57 \mathrm{~nm}$ for the fibers in absence of graphene to $141 \mathrm{~nm}$ for a 2 wt. (\%) of graphene added. It should be stressed, however, that the average diameters were $37 \%$ smaller than the values reported in the literature.
\end{abstract}

Keywords: Electrospinning, nanofibers, nanomembranes, graphene, nanostructured composites.

\section{Introdução}

Devido à elevada relação entre sua área superficial e seu volume, à interconectividade de suas fibras e à existência de espaço intertistial, as nanomembranas são de grande interesse em um grande número de aplicações onde estruturas porosas são desejáveis, tais como: compósitos estruturais, exocompósitos, nanobiotecnologia, sistemas de purificação de ar, bioengenharia, engenharia ambiental e eletrônica, e indústrias energética, de defesa e segurança ${ }^{[1-5]}$.

Embora a técnica de fabricação de fibras sintéticas utilizando descargas elétricas tenha sido descoberta no século passado ${ }^{[6,7]}$, foi apenas recentemente que o electrospinning tornou-se alvo de um grande número de pesquisadores em todo o mundo ${ }^{[8,9]}$. O princípio operacional do processo de electrospinning é relativamente simples, tornando-o bastante vantajoso quando comparado aos demais métodos de fabricação de nanofibras ${ }^{[2]}$. Além disso, o controle dimensional das nanofibras geradas, a reprodutibilidade de resultados e o grande potencial para aplicações industriais em larga escala fizeram com que o processo de electrospinning se tornasse bastante "popular" nos últimos anos.

A técnica de electrospinning envolve a aplicação de um forte campo elétrico entre o polímero e um coletor metálico ${ }^{[3,10,11]}$. Geralmente, o polímero, fundido ou em solução, está contido em um reservatório com um tubo capilar (tipicamente, uma seringa hipodérmica e uma agulha), e é forçado a escoar por uma agulha por ação da gravidade ou com o auxílio de uma bomba de infusão. Quando se aplica uma alta tensão elétrica no tubo capilar, que está localizado à uma certa distância de um coletor (que pode ser plano e estacionário ou cilíndrico e rotativo), ocorre uma polarização nas moléculas do polímero. Quando a diferença de potencial elétrico entre o tubo capilar e o coletor ultrapassa um valor crítico, a força eletrostática atuando no polímero passa a ser maior do que a tensão interfacial da solução na ponta do tubo, formando o chamado cone de Taylor e acarretando a formação de um jato que é atraído em direção ao coletor. Neste ponto, o campo elétrico é responsável por alongar o jato, tornando-o cada vez mais fino, dando origem às fibras de diâmetros micro ou nanométricos ${ }^{[12,13]}$.

Para que o processo de electrospinning seja possível, é necessário que o polímero esteja fundido ou disperso em solução. Dessa forma, fica evidente que as propriedades da solução (peso molecular do polímero, viscosidade, tensão interfacial e condutividade elétrica da solução, e efeito dielétrico do solvente) irão desempenhar um papel importante no processo de geração das nanofibras. Outros fatores de grande influência

Autor para correspondência: Antônio F. Ávila, Departamento de Engenharia Mecânica, Universidade Federal de Minas Gerais - UFMG, CEP 31270-901, Belo Horizonte, MG, Brasil, e-mail: aavila@netuno.lcc.ufmg.br 
no electrospinning são relacionados aos parâmetros de operação do processo propriamento dito. Dentre estes fatores, pode-se citar a diferença de potencial utilizada para se gerar o campo elétrico, a vazão da solução, a temperatura, o tipo de coletor utilizado, o diâmetro do orifício da seringa e a distância entre a seringa e o coletor. Finalmente, os fatores ambientais, tais como a umidade relativa do ar, a pressão e a composição da atmosfera (utilização de gases diferentes), também podem influenciar nos resultados obtidos pelo processo de electrospinning ${ }^{[2,14]}$.

Um parametro adicional que pode influenciar a morfologia das nanomembranas geradas por electrospinning é a adição de nanopartículas na solução polimérica. Nanotubos de carbono, nanofolhas de grafeno, nanopartículas de prata, ouro e sílica coloidal, e nanocerâmicas estão entre as nanopartículas mais utilizadas atualmente. O grafeno, que possui propriedades mecânicas, térmicas e elétricas similares às dos nanotubos de carbono, são 500 vezes mais baratos e estão se tornando uma alternativa bastante promissora $^{[15-18]}$.

Devido às suas excelentes propriedades físicas, químicas e térmicas ${ }^{[5]}$, o polímero utilizado no estudo dos parâmetros de operação do processo de electrospinning foi a poliamida-66 (PA-66), também conhecido com Nylon-66 ${ }^{\circledR}$. Como solvente, optou-se pela utilização de uma mistura contendo $75 \%$ de ácido fórmico $\left(\mathrm{CH}_{2} \mathrm{O}_{2}\right)$ e $25 \%$ de clorofórmio $\left(\mathrm{CHCl}_{3}\right)^{[19]}$.

\section{Experimental}

\section{Materiais}

O polímero poliamida-66 (Nylon-66 $6^{\circledR}$ ) com peso molecular de $226,32 \mathrm{~g} \cdot \mathrm{mol}^{-1}$ foi fornecido pela Rhodia. O ácido fórmico $\left(\mathrm{CH}_{2} \mathrm{O}_{2}\right)$ e o clorofórmio $\left(\mathrm{CHCl}_{3}\right)$ foram obtidos junto à $\mathrm{Synth}^{\circledR}$. O grafite expandido HC 11-IQ foi fornecido pela Nacional Grafite. Todos os materiais foram utilizadas como adquiridos.

\section{Electrospinning}

O electrospinning foi realizado com o auxílio da unidade de electrospinning do "Laboratório de Mecânica de Compósitos" da UFMG. Dentre os vários parâmetros de operação disponíveis, optou-se por variar apenas aqueles que tem maior influência na morfologia das fibras ${ }^{[20]}$, ou seja: a concentração do polímero na solução, a diferença de potencial elétrico aplicada, a vazão da solução, a distância entre a ponta da agulha e o coletor e a concentração de nanopartículas na solução polimérica. A velocidade de deslocamento transversal do suporte da seringa e a rotação do coletor foram fixadas em $6,25 \mathrm{~cm} / \mathrm{min}$ e $19 \mathrm{rpm}$, respectivamente.

Baseando-se nos resultados obtidos na literatura ${ }^{[5,19,21-23]}$, foram geradas nanomembranas de PA-66 conforme dados da Tabela 1.

As soluções utilizadas no electrospinning foram obtidas através da dissolução de PA-66 em ácido fórmico/clorofórmio (75\%:35\%) por 1 hora e 30 minutos à temperatura ambiente, utilizando um misturador magnético IKA ${ }^{\circledR}$ CERAMAG Midi a 600 rpm. Após este período, foram adicionadas as nanofolhas de grafeno originadas do grafite expandido HC 11-IQ às soluções X a XV que, novamente, voltaram ao misturador magnético por mais 1 hora e 30 minutos a $600 \mathrm{rpm}$. Uma vez homogeneizadas, as soluções poliméricas "repousaram" por 1 hora, para que todas as bolhas de ar, criadas durante o processo de mistura, fossem liberadas. Em seguida, $10 \mathrm{~mL}$ das soluções poliméricas foram colocadas em seringas hipodérmicas de $20 \mathrm{~mL}$ com agulhas de aço inoxidável com ponta G18 (diâmetros interno e externo de $0,84 \mathrm{~mm}$ e $1,27 \mathrm{~mm}$, respectivamente), para serem utilizadas no processo de electrospinning.

As nanomembranas foram depositadas por 2 horas sobre o coletor cilíndrico da unidade de electrospinning, que foi coberto por papel alumínio para facilitar a remoção das nanomembranas geradas. O processo de secagem das nanomembranas se deu em uma estufa a vácuo Quimis Q-819V2 por um período de 2 horas à uma temperatura constante de $70{ }^{\circ} \mathrm{C}$.

\section{Caracterização das soluções poliméricas}

A caracterização das soluções poliméricas utilizadas no electrospinning foi realizada por meio da análise de viscosidade dinâmica utilizando um viscosímetro de cilindro rotativo Cole-Parmer ${ }^{\circledR}$ EW-98936 à $25^{\circ} \mathrm{C}$ e com rotação de $0,3 \mathrm{rpm}$. As medidas de condutividade foram realizadas com o uso de um condutivimetro Hanna HI98311.

\section{Caracterização morfológica}

A caracterização morfológica das nanomembranas geradas foi realizada por meio de microscopia eletrônica de varredura (MEV) utilizando um microscópio $\mathrm{FEI}^{\circledR}$ Quanta 200-FEG. As amostras de nanomembranas foram montadas em stubs de alumínio e recobertas com uma camada de ouro $(1 \mathrm{~nm})$, por meio do Bal-Tec/ Leica MED 020 Coating System, para se evitar o carregamento das imagens durante a varredura do microscópio. Utilizando-se

Tabela 1. Parâmetros utilizados na fabricação de nanomembranas por electrospinning.

\begin{tabular}{|c|c|c|c|c|c|}
\hline Amostra & $\begin{array}{c}\text { Concentração do } \\
\text { polímero (\% em peso) }\end{array}$ & $\begin{array}{c}\text { Dif. de potencial } \\
\text { elétrico }(\mathrm{kV})\end{array}$ & $\begin{array}{c}\text { Vazão da solução } \\
(\mathrm{mL} / \mathrm{h})\end{array}$ & $\begin{array}{l}\text { Distância agulha- } \\
\text { coletor }(\mathbf{c m})\end{array}$ & $\begin{array}{c}\text { Concentração de } \\
\text { grafeno }(\% \text { em peso })\end{array}$ \\
\hline I & 10 & 25 & 0,17 & 15 & 0 \\
\hline II & 12 & 25 & 0,17 & 15 & 0 \\
\hline III & 15 & 25 & 0,17 & 15 & 0 \\
\hline IV & 10 & 20 & 0,17 & 15 & 0 \\
\hline $\mathrm{V}$ & 10 & 22,5 & 0,17 & 15 & 0 \\
\hline VI & 10 & 25 & 0,34 & 15 & 0 \\
\hline VII & 10 & 25 & 0,51 & 15 & 0 \\
\hline VIII & 10 & 25 & 0,17 & 10 & 0 \\
\hline IX & 10 & 25 & 0,17 & 12,5 & 0 \\
\hline $\mathrm{X}$ & 10 & 25 & 0,17 & 15 & 1 \\
\hline XI & 10 & 25 & 0,17 & 15 & 2 \\
\hline XII & 12 & 25 & 0,17 & 15 & 1 \\
\hline XIII & 12 & 25 & 0,17 & 15 & 2 \\
\hline XIV & 15 & 25 & 0,17 & 15 & 1 \\
\hline XV & 15 & 25 & 0,17 & 15 & 2 \\
\hline
\end{tabular}


o software de análise de imagens Image ${ }^{[24]}$, determinou-se os diâmetros médios das fibras de todas as nanomembranas. Para tanto, foram realizadas 50 medições de diâmetros nas imagens MEV de cada amostra.

Para a análise de difração de raios $\mathrm{X}$ foi utilizado um difratometro Shimadzu XRD-6000 X-com irradiação com filamento de cobre $(\lambda=0.154 \mathrm{~nm})$ a $40 \mathrm{kV}$ e $30 \mathrm{~mA}$ usando um filtro de Ni. A coleta de dados foi realizada a cada $2^{\circ}$ até $60^{\circ}$.

\section{Resultados e Discussões}

Os valores médios de viscosidade dinâmica das soluções poliméricas podem ser vistos na Tabela 2. Comparando os valores obtidos para as soluções poliméricas sem carregamento de grafeno, observa-se que a viscosidade dinâmica mantem uma relação de alometria $(\mu \propto C \beta)$ com a concentração de polímero na solução, conforme observado por He et al. ${ }^{[25]}$. Entretanto, com o aumento

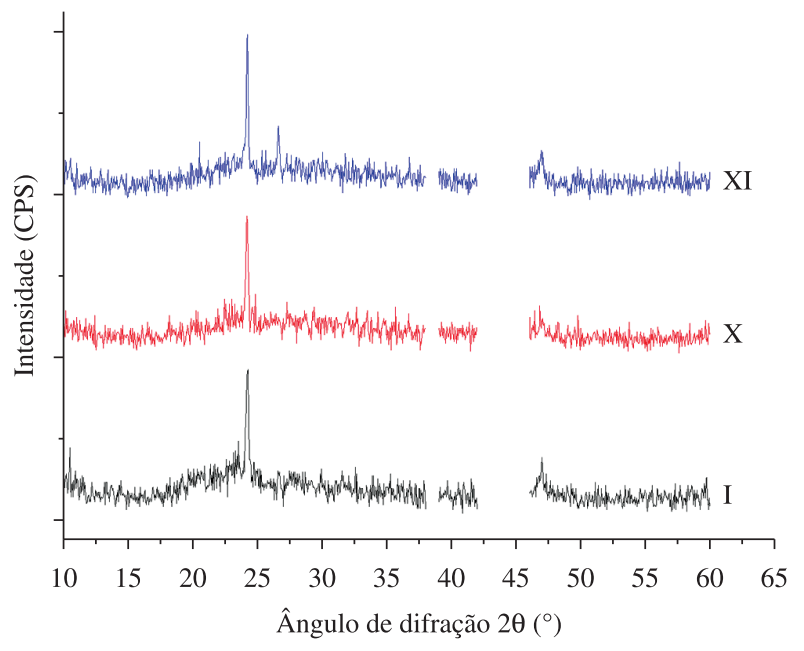

(b) (a)

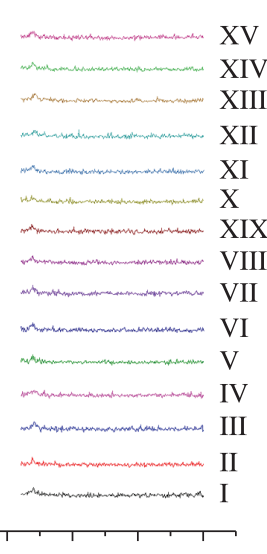

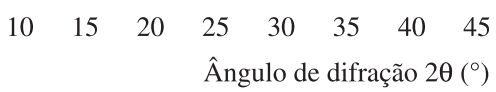

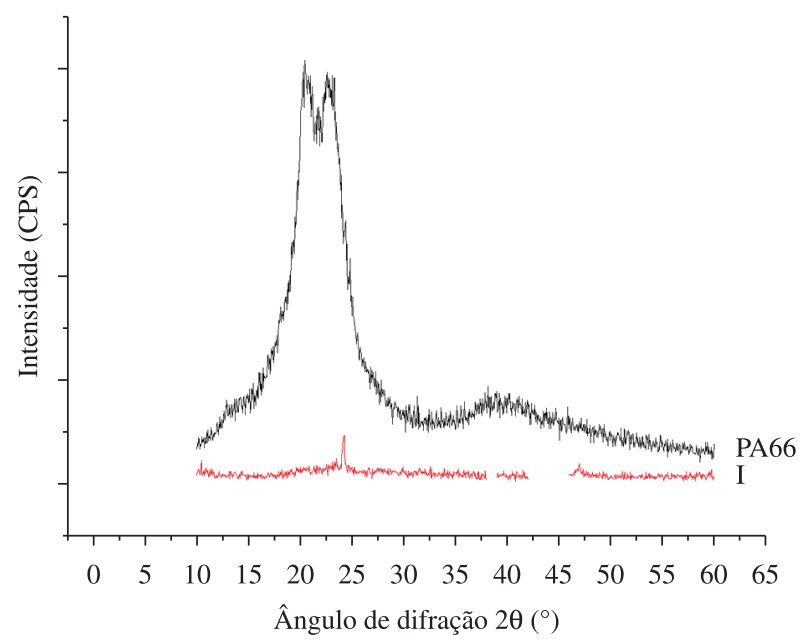

(c)

Figura 1. Difratograma das nanomembranas. a) Amostras de I a XV; b) Zoom nos difratogramas das amostras I, X e XI; c) Amostra de PA 66 puro e amostra I.

Tabela 2. Viscosidade dinâmica média das soluções poliméricas utilizadas no electrospinning.

\begin{tabular}{cccc}
\hline $\begin{array}{c}\text { Concentração do polímero } \\
\text { (\% em peso) }\end{array}$ & $\begin{array}{c}\text { Concentração de grafeno } \\
\text { (\% em peso) }\end{array}$ & $\begin{array}{c}\text { Viscosidade dinâmica } \\
(\mathbf{m P a} . \mathbf{s})\end{array}$ & Amostras \\
\hline 10 & 0 & 260 & $\mathrm{I}$, IV, V, VI, VII, VIII, IX \\
10 & 1 & 401 & $\mathrm{X}$ \\
10 & 2 & 955 & $\mathrm{XI}$ \\
12 & 0 & 483 & $\mathrm{II}$ \\
12 & 1 & 1992 & $\mathrm{XII}$ \\
12 & 2 & 3127 & $\mathrm{XIII}$ \\
15 & 0 & 2733 & $\mathrm{III}$ \\
15 & 1 & 4520 & $\mathrm{XIV}$ \\
\hline
\end{tabular}


do carregamento de grafeno para soluções poliméricas com mesma concentração de polímero, o aumento da viscosidade dinâmica foi aproximadamente linear.

A Figura 1 mostra o padrão de difração de raios X para os 15 grupos estudados. Observa-se que a presença de grafeno $\left(\right.$ pico $\approx 26,8^{\circ}$ ) pode ser detectadas nas amostras XI, XIII e XV. Estes picos, evidenciam a formação de nano-estruturas de nanofolhas de grafeno na forma intercalada nas nanomembranas. As diferenças na concentração de grafeno também podem ser observadas através da difração de raios X (veja Figura 1).

Guerrini et al. ${ }^{[26]}$, utilizou a calorimetria diferencial exploratória (DSC) para determinação do grau de cristalinidade das nanomembranas.

Tabela 3. Diâmetros médios das nanofibras das amostras geradas por electrospinning, RIR e condutividade elétrica.

\begin{tabular}{|c|c|c|c|c|c|c|}
\hline Amostra & No de medidas & Média (nm) & Desvio padrão (nm) & Coef. Variação (\%) & $\operatorname{RIR}(\%)$ & $\mathrm{K}\left(\mathbf{m S} . \mathrm{cm}^{-1}\right)$ \\
\hline I & 48 & 57 & 15 & 25,7 & 11,4 & 4.2 \\
\hline II & 46 & 98 & 23 & 23,2 & 13,0 & 4.3 \\
\hline IV & 48 & 49 & 11 & 21,3 & 12,1 & 4.2 \\
\hline $\mathrm{V}$ & 46 & 54 & 15 & 27,4 & 12,0 & 4.2 \\
\hline VI & 47 & 69 & 17 & 24,9 & 13,5 & 4.2 \\
\hline VII & 50 & 81 & 28 & 34,8 & 11,5 & --- \\
\hline VIII & 48 & 52 & 12 & 22,7 & 11,5 & --- \\
\hline IX & 50 & 50 & 13 & 26,2 & 11,0 & --- \\
\hline$X$ & 46 & 72 & 19 & 27,2 & 10,9 & --- \\
\hline $\mathrm{XI}$ & 48 & 57 & 9 & 16,5 & 13,0 & 3.6 \\
\hline XII & 49 & 67 & 15 & 23,0 & 13,4 & 3.4 \\
\hline XIV & 43 & 137 & 37 & 27,0 & 11,7 & -- \\
\hline XV & 50 & 141 & 44 & 31,1 & 10,9 & -- \\
\hline
\end{tabular}

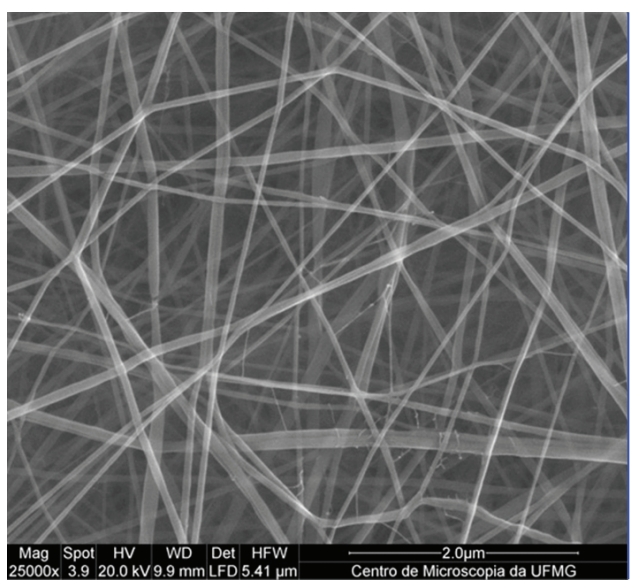

(a)

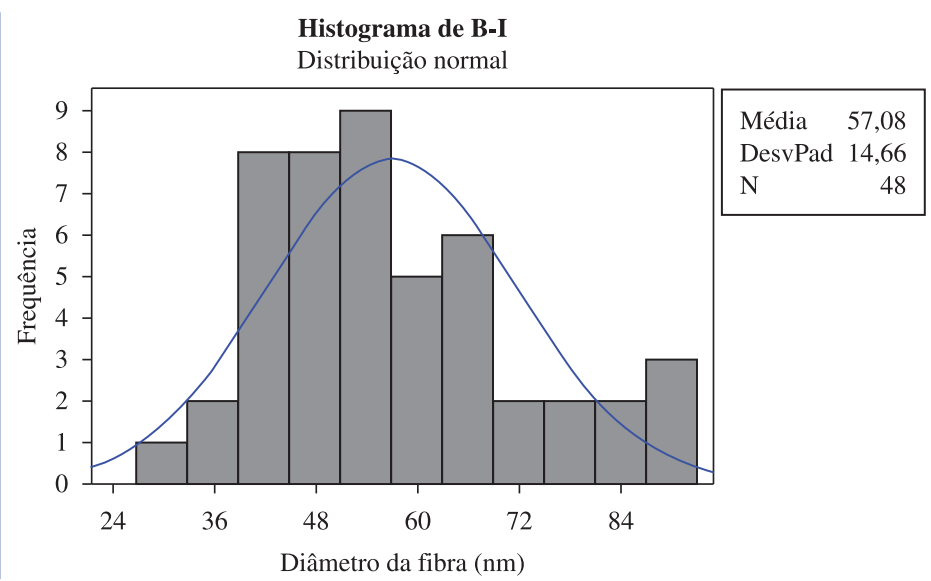

(b)

Figura 2. Morfologia das fibras da amostra I: a) imagem MEV (25.000×); b) distribuição dos diâmetros encontrados.

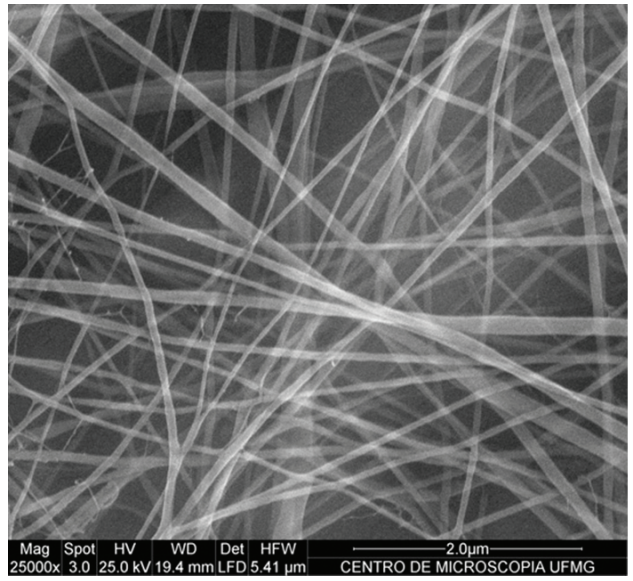

(a)

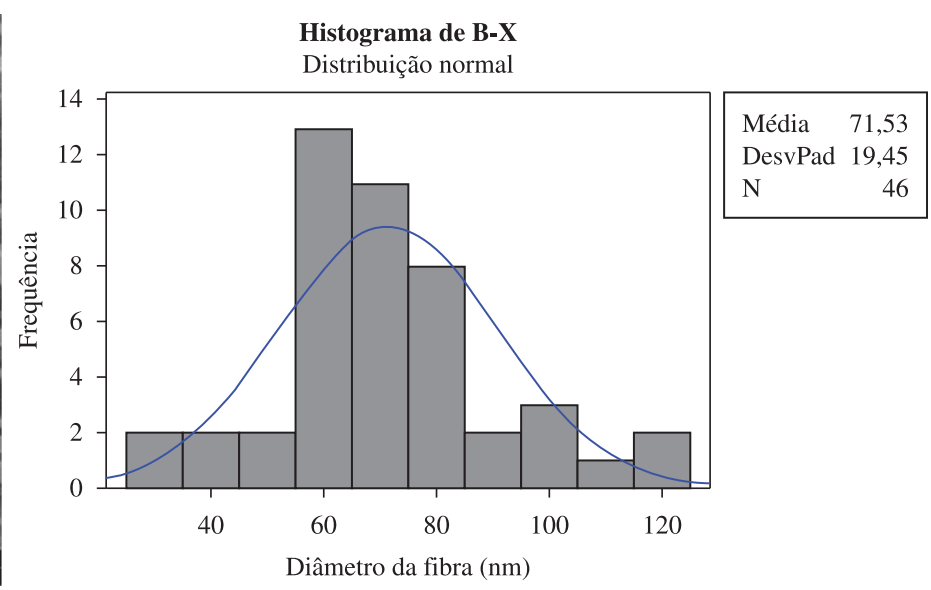

(b)

Figura 3. Morfologia das fibras da amostra X: a) imagem MEV (25.000x); b) distribuição dos diâmetros encontrados. 
No entanto, como mencionado por Guerrini et al. ${ }^{[9]}$, uma outra possibilidade para o cálculo da cristalinidade é o uso de difração de raios X (DRX). Para tal foi utilizado o índice de razão de intensidade (RIR) como definido por Prevéy ${ }^{[27]}$. A Tabela 3 resume os resultados obtidos. Note que os resultados reportados por Guerrini et al. ${ }^{[26]}$ apresentam uma variação de cristalinidade entre $15 \%$ e $30 \%$, enquanto que os resultados reportados neste estudo estão entre 10,9\% e 13,5\%. As diferenças nos resultados deve-se prinicipalmente nas diferentes concentrações dos polímeros (Guerrini variou entre 15 e 20\%), os diferentes campos elétricos aplicados e as técnicas utilizadas (DXR e DSC). Quando comparados resultados para a mesma concentração de PA 66 (15\%) Guerrini et al. ${ }^{[26]}$ reportaram $15 \%$ enquanto que a amostra III indica $12,5 \%$. Diferentemente dos resultados apresentados por Guerrini, onde a "assinatura" de DRX das amostras indicaram um comportamento quasi-amorfo, os resultados apresentados nesta pesquisa detectaram a formação de estruturas cristalinas (veja Figura 1).

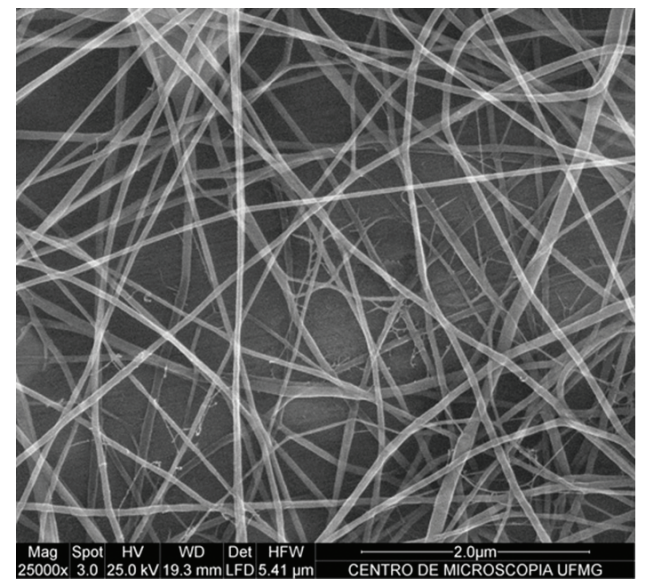

(a)

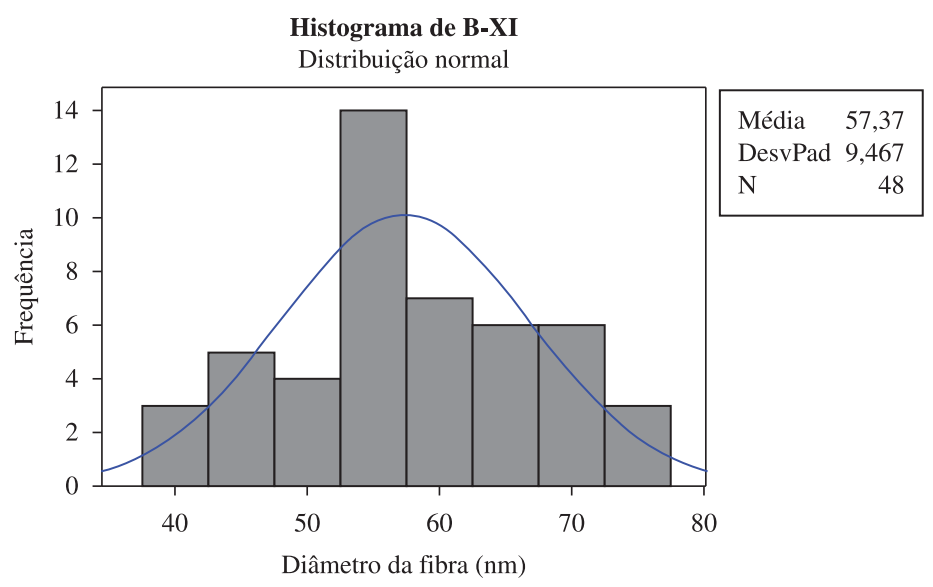

(b)

Figura 4. Morfologia das fibras da amostra XI: a) imagem MEV (25.000x); b) distribuição dos diâmetros encontrados.

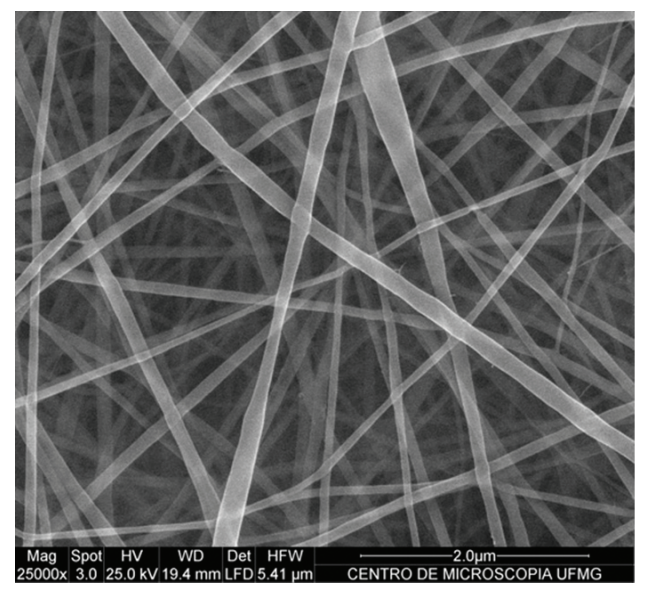

(a)

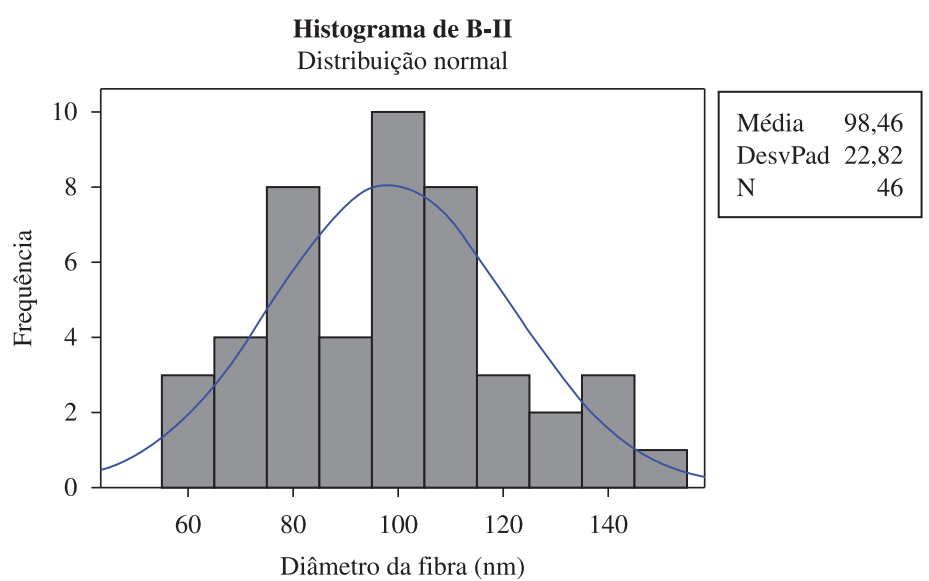

(b)

Figura 5. Morfologia das fibras da amostra II: a) imagem MEV (25.000x); b) distribuição dos diâmetros encontrados.

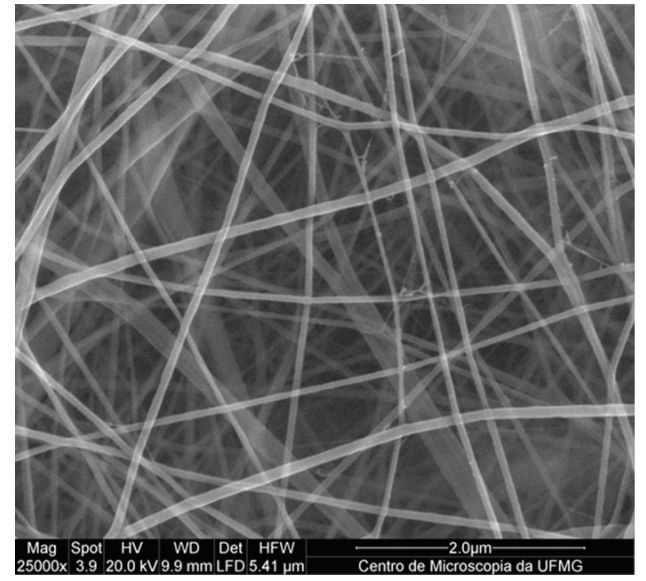

(a)

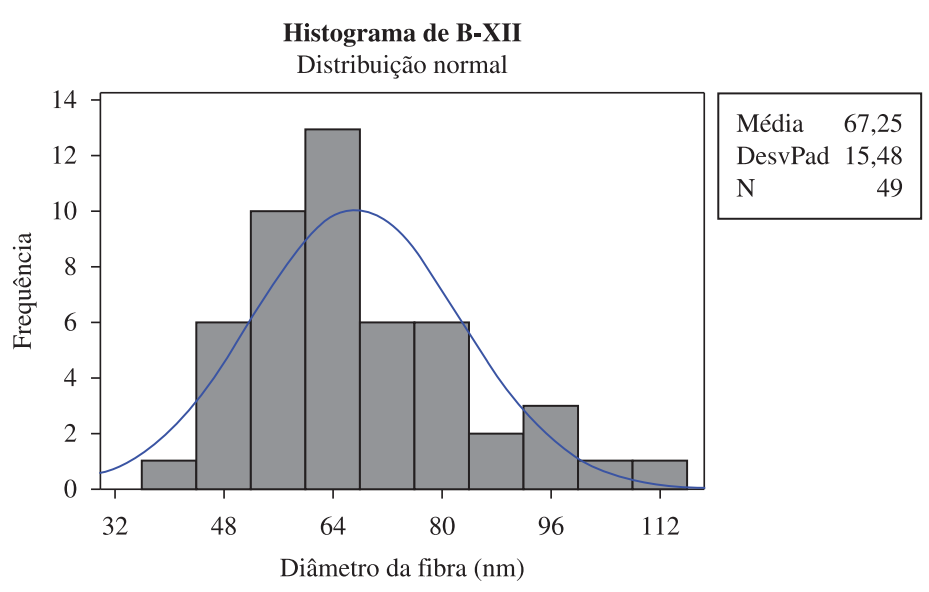

(b)

Figura 6. Morfologia das fibras da amostra XII: a) imagem MEV (25.000x); b) distribuição dos diâmetros encontrados. 
O difractograma de PA 66 evidência claramente a natureza cristalina deste material com a fase $\alpha(010)$ em torno de $23^{\circ}$. A mesma fase $\alpha$ pode ser observada na amostra I, com uma intensidade muito menor. Mais ainda, a adição de nanofolhas de grafeno gerou nano-estruturas de diâmetros de aproximadamente $0.31 \mathrm{~nm}$ e $0.41 \mathrm{~nm}$ para as amostras X e XI, respectivamente.

As medições de condutividade das soluções foram realizadas a temperatura ambiente $\left(25^{\circ} \mathrm{C}\right)$ e a umidade relativa de $50 \%$. Os valores apresentados na Tabela 3 são as médias de 3 medições realizadas. Os valores encontrados variam entre 4.2 e 4.3 dependendo da concentração de PA 66, resultados semelhantes foram reportados por Guerrini et al. ${ }^{[26]}$. Devido a natureza corrosiva das soluções algumas medições não foram realizadas. Os resultados entre 3.6 e $2.9 \mathrm{mS} . \mathrm{cm}^{-1}$ podem ser atribuídos a corrosão dos eletrodos e consequente perda de precisão do instrumento. Guerrini et al. ${ }^{[26]}$ também não forneceram todos os resultados, provavelmente devido ao mesmo problema.

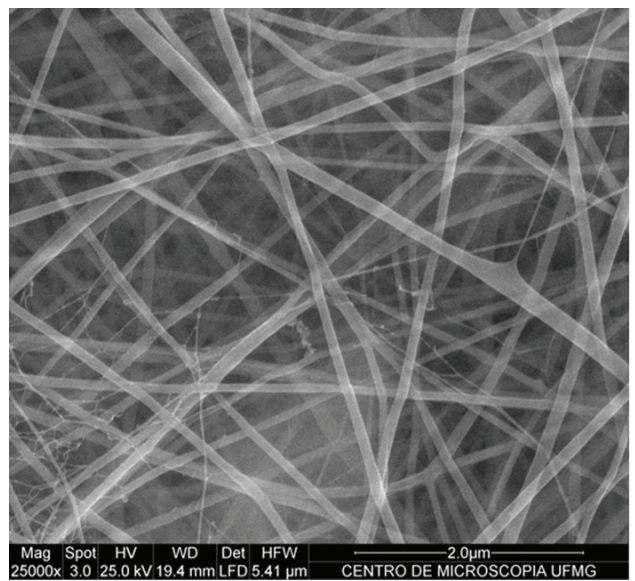

(a)

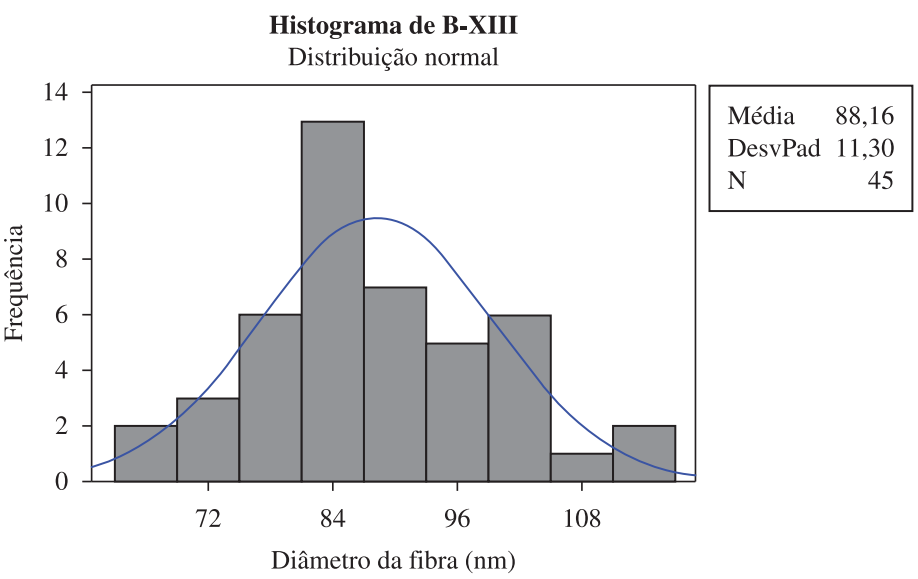

(b)

Figura 7. Morfologia das fibras da amostra XIII: a) imagem MEV (25.000×); b) distribuição dos diâmetros encontrados.

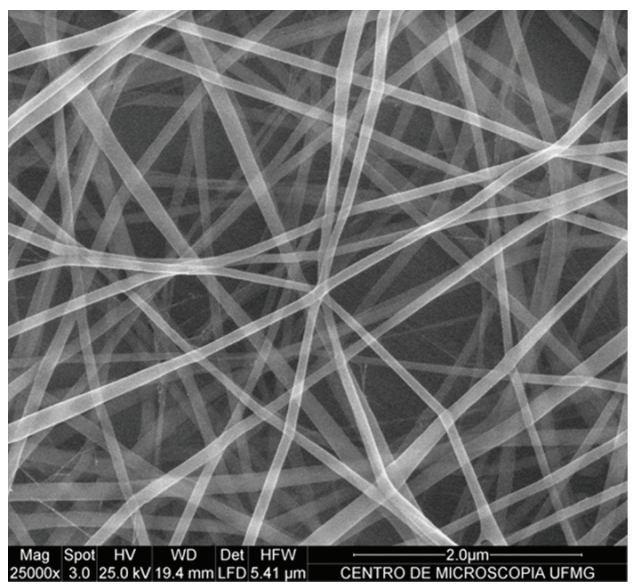

(a)

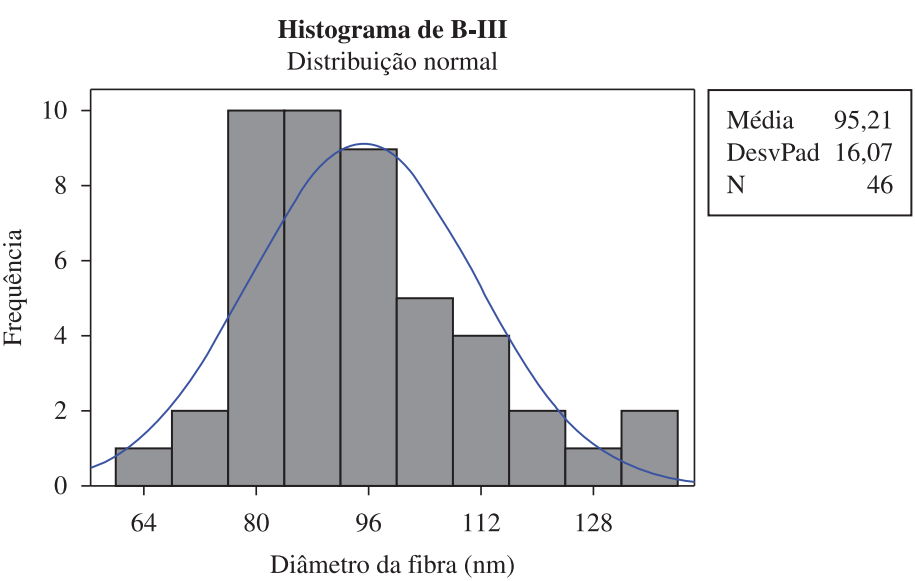

(b)

Figura 8. Morfologia das fibras da amostra III: a) imagem MEV (25.000×); b) distribuição dos diâmetros encontrados.

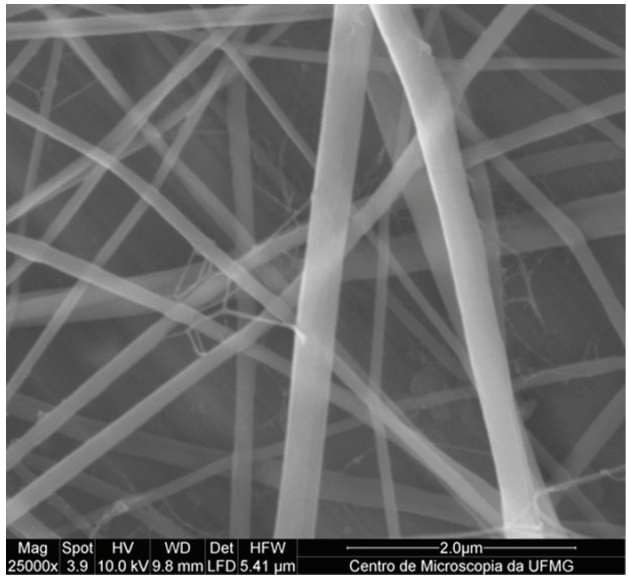

(a)

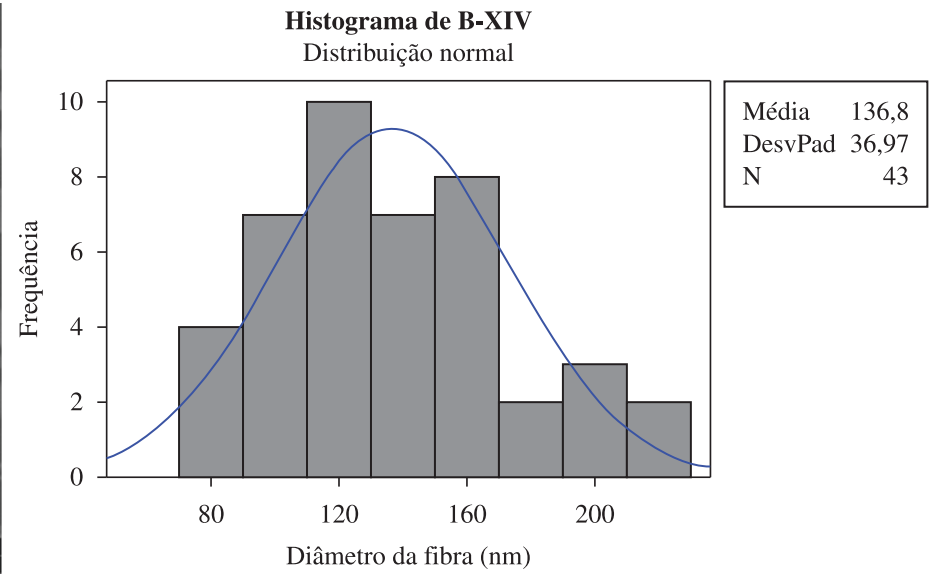

(b)

Figura 9. Morfologia das fibras da amostra XIV: a) imagem MEV (25.000×); b) distribuição dos diâmetros encontrados. 


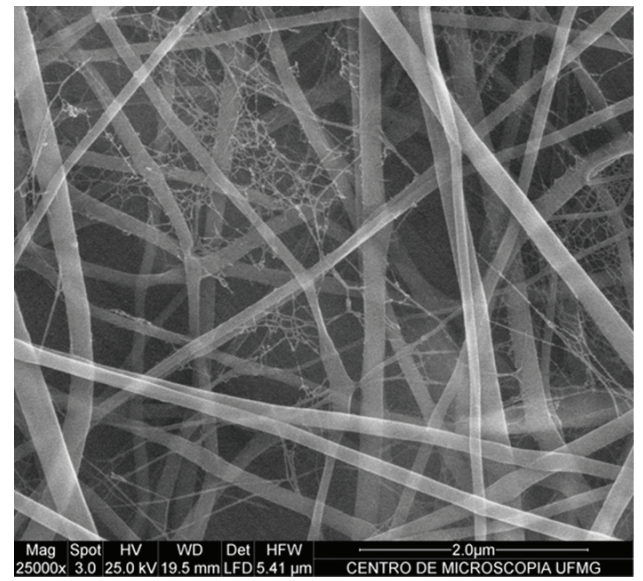

(a)

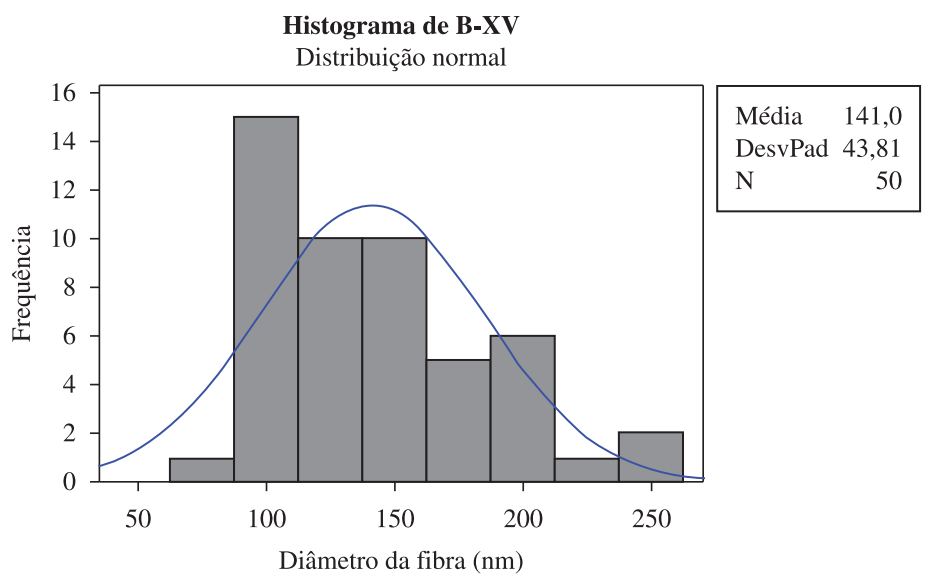

(b)

Figura 10. Morfologia das fibras da amostra XV: a) imagem MEV (25.000x); b) distribuição dos diâmetros encontrados.

Os valores médios de diâmetros das nanofibras obtidas, encontrados para cada amostra, podem ser vistos na Tabela 3, após a eliminaçãoo dos outliers ou dados inconsistentes ou valores extremos que diferem muito do comportamento dos dados restantes.

A Figuras 2-10 mostram imagens MEV de cada amostra, bem como a distribuição de diâmetros encontrados nas nanomembranas produzidas, para as configurações I, X, XI (para a concentração de $10 \%$ de PA-66), II, XII, XII (12\% de PA-66) e III, XIV, XV (15\% de PA-66), respectivamente.

\section{Influência da concentração do polímero na solução polimérica}

Comparando independentemente as amostras II e III com a amostra I (controle), observou-se que houve um aumento estatisticamente significativo $(\mathrm{P}=0,000<0,05$, segundo uma análise de variância) do diâmetro médio das fibras com o aumento da concentração de PA-66 na solução polimérica, o que está em concordância com os resultados experimentais encontrados na literatura $^{[19,28,29]}$.

Digno de nota é o fato de que os diâmetros médios das nanofibras das amostras I e II são muito menores que os valores obtidos por Lingaiah et al. ${ }^{[19]}(60-120 \mathrm{~nm}$ e $120-240 \mathrm{~nm}$ para $10 \%$ e $12 \%$ de PA-66, respectivamente) para o mesmo polímero.

\section{Influência da diferença de potencial elétrico aplicada}

Comparando as amostras IV e V com a amostra I (controle), observou-se que houve um aumento estatisticamente significativo $(\mathrm{P}=0,019<0,05)$ do diâmetro médio das fibras com o aumento da diferença de potencial elétrico aplicada, conforme observado por Li et al. ${ }^{[29]}$.

\section{Influência da vazão da solução}

Comparando as amostras VI e VII com a amostra I (controle), observou-se que houve um aumento estatisticamente significativo $(\mathrm{P}=0,000<0,05)$ e contínuo do diâmetro médio das fibras com o aumento da vazão da solução polimérica. Tais resultados estão de acordo com o comportamento descrito na teoria ${ }^{[2]}$.

\section{Influência da distância entre a agulha e o coletor}

Comparando as amostras VIII e IX com a amostra I (controle), observou-se que houve uma variação estatisticamente significativa $(\mathrm{P}=0,028<0,05)$ do diâmetro médio das fibras. Conforme descrito por Ramakrishna et al. ${ }^{[2]}$, os resultados comprovam que a diminuição da distância entre a agulha e o coletor aumenta a intensidade do campo elétrico, que é responsável por alongar as nanofibras durante o processo de deposição, ocasionando a diminuição do diâmetro médio das fibras.

\section{Influência da concentração de grafeno na solução polimérica}

Comparando a amostra X com a amostra I (controle), observou-se que houve um aumento significativo $(\mathrm{P}=0,000<0,05)$ do diâmetro médio das fibras, que passou de $57 \mathrm{~nm}$ para $72 \mathrm{~nm}$, conforme os resultados experimentais apresentados por Wang et al. ${ }^{[30]}$ para outras nanopartículas. Comparando a amostra XI com a amostra I (controle), observou-se que não houve uma variação estatisticamente significativa $(\mathrm{P}=0,908>0,05)$ do diâmetro médio das fibras, o que pode ter sido causado pelo entupimento parcial e temporário da agulha durante o processo de deposição via electrospinning, gerando fibras mais finas que o esperado.

Comparando as amostras XII e XIII com a amostra II (controle), observou-se que não houve uma variação coerente do diâmetro médio das fibras com o aumento da concentração de nanofolhas de grafeno para solução polimérica contendo 12\% de PA-66. Embora não tenha havido uma redução significativa no número de nanofibras depositadas, a variação de resultados aqui apresentada pode ter sido causada pelo fato de que estas amostras foram as que mais tiveram problemas com entupimento parcial/temporário da agulha. Provavelmente, esse comportamento foi causado pela mudança no ambiente em que estava sendo realizado o processo de deposição das fibras por electrospinning. Entretanto, não houve uma diferença estatisticamente significativa entre os resultados.

Comparando as amostras XIV e XV com a amostra III (controle), observou-se que houve um aumento significativo $(\mathrm{P}=0,000<0,05)$ e contínuo do diâmetro médio das fibras com o aumento da concentração de grafeno para solução polimérica contendo $15 \%$ de PA-66. Tais resultados estão de acordo com o comportamento descrito por Ramakrishna et al. ${ }^{[2]}$.

\section{Conclusões}

O processo de electrospinning e sua influência na morfologia das nanofibras formadas, foi avaliado utilizado a poliamida-66 (PA-66) com e sem carregamento de grafeno. Os resultados obtidos mostraram que os seguintes parâmetros operacionais da técnica de electrospinning são proporcionais ao diâmetro das nanofibras: diferença de potencial elétrico aplicada, vazão da solução polimérica 
e concentração do polímero na solução. Os resultados também mostraram que a distância entre o coletor e o emissor (ponta da agulha) é inversamente proporcional ao diâmetro da nanofibra formada. Os diâmetros das nanofibras preparadas apresentaram uma distribuição quasi-normal e variavam entre $49 \mathrm{~nm}$ e $141 \mathrm{~nm}$. Tais valores são $37 \%$ menores que aqueles reportados na literatura.

A adição de nanofolhas de grafeno, nas proporções $1 \%$ e $2 \%$ em peso, às soluções de PA-66 fez com que alterações nos diâmetros fossem observadas. Para a concentração de $10 \%$ de PA-66, a adição de $1 \%$ em peso de grafeno resultou em um aumento do diâmetro médio de $57 \mathrm{~nm}$ para $72 \mathrm{~nm}$, que já era esperado. No entanto, a adição de $2 \%$ de grafeno não resultou em aumento significativo no diâmetro. Pelo contrário, o diâmetro se manteve estável em torno de $57 \mathrm{~nm}$. Uma possível explicação é o entupimento parcial/ temporário do orifício da agulha, o que fez com que o fluxo de solução polimérica reduzisse e, com isso, o efeito da adição das nanopartículas foi praticamente neutralizado. Para a concentração de 12\% de PA-66 foi observada a redução do diâmento médio das nanofibras de $98 \mathrm{~nm}$ para $67 \mathrm{~nm}$ e $88 \mathrm{~nm}$ para as concentrações de $1 \%$ e $2 \%$, respectivamente. Neste caso, o aumento da viscosidade fez com que a adição de nanopartículas de grafeno causasse uma influência secundária. Para a concentração de 15\% de PA-66 da solução polimérica, a adição de grafeno resultou em um aumento do diâmetro de $95 \mathrm{~nm}$ para $137 \mathrm{~nm}$ e $141 \mathrm{~nm}$ para $1 \%$ e $2 \%$, respectivamente. $\mathrm{O}$ aumento da concentração de nanopartículas de base carbono parece não ter sido um fator importante, pois o aumento de $1 \%$ para $2 \%$ de nanofolhas de grafeno fez com que houvesse uma variação no diâmetro de apenas $5 \%$ em relação ao diâmetro original. $\mathrm{O}$ fator preponderante neste caso parece ter sido o aumento da viscosidade dinâmica causada pelo aumento da concentração do PA-66 na solução.

\section{Agradecimentos}

Os autores gostariam de agradecem ao Programa de Pós-Graduação em Engenharia Mecânica da UFMG, à CAPES, ao Air Force Office of Scientific Research (AFOSR) projeto FA9550-101-0050, a FAPEMIG projeto PPM-00192-12 pelo apoio acadêmico e financeiro, e ao Centro de Microscopia da UFMG, por prover os equipamentos e o suporte técnico necessário para os experimentos envolvendo microscopia eletrônica.

\section{Referências Bibliográficas}

1. Huang, Z.-M.; Zhang, Y.-Z.; Kotaki, M. \& Ramakrishna, S. - Comp. Sci. Technol., 63, p.2223 (2003). http://dx.doi.org/10.1016/S02663538(03)00178-7

2. Ramakrishna, S.; Fujihara, K.; Teo, W.-E.; Lim, T.-C. \& Ma, Z. - "An Introduction to Electrospinning and Nanofibers", World Scientific, Singapore (2005).

3. Andrady, A. L. - "Science and Technology of Polymer Nanofibers", John Wiley, New Jersey (2008). http://dx.doi.org/10.1002/9780470229842

4. Li, G.; Li, P.; Yu, Y.; Jia, X.; Zhang, S.; Yang, X. \& Ryu, S. - Mat. Lett., 62, p.511 (2008). http://dx.doi.org/10.1016/j.matlet.2007.05.080

5. Shivakumar, K.; Lingaiah, S.; Chen, H.; Akangah, P.; Swaminathan, G.; Sharpe, M. \& Sadler, R. - "Polymer nanofabric interleaved composite laminates", in: Proceedings of 50th AIAA/ASME/ASCE/ AHS/ASC Structures, Structural Dynamics and Materials Conference, p.1, Palm Springs - USA, maio (2009).

6. Cooley, J. F. - "Apparatus for Electrically Dispersing Fluids", US Patent 692.631 (1902).

7. Formhals, A. - "Process and Apparatus for Preparing Artificial Threads", US Patent 1.975.504 (1934).

8. Ko, F. K. - "Nanofiber technology: Bridging the gap between nano and macro world", in: Nanoengineered Nanofibrous Materials, cap.1.1,
S. Guceri; Y. G. Gogotsi \& V. Kuznetsov (eds.), Kluwer Academic Publishers, Dordrecht, Netherlands (2004).

9. Guerrini, L. M.; Branciforti, M. C.; Bretas, R. E. S. \& De Oliveira, M. P. - Polímeros, 16, p.286, 2006.

10. Norris, I. D.; Shaker, M. M.; Ko, F. K. \& Macdiarmid, A. G. - Synthetic Met., 114, p.109 (2000). http://dx.doi.org/10.1016/ S0379-6779(00)00217-4

11. Ayutsede, J. E. - "Regeneration of Bombyx Mori Silk Nanofibers and Nanocomposite Fibrils by The Electrospinning Process". Tese de Doutorado, Drexel University, USA (2005).

12. Lam, H. L. - "Electrospinning of Single Wall Carbon Nanotube Reinforced Aligned Fibrils and Yarns", Tese de Doutorado, Drexel University, USA (2004).

13. Doshi, J. \& Reneker, D. H. - J. Electrost., 35, p.151 (1995). http:// dx.doi.org/10.1016/0304-3886(95)00041-8

14. Reneker, D. H. \& Yarin, A. L. - Polymer, 49, p.2387 (2008). http:// dx.doi.org/10.1016/j.polymer.2008.02.002

15. Yasmin, A. \& Daniel, I. M. - Polymer, 45, p.8211 (2004). http://dx.doi. org/10.1016/j.polymer.2004.09.054

16. Mack, J. J.; Viculis, L. M.; Ali, A.; Luoh, R.; Yang, G.; Hahn, H. T.; Ko, F. K. \& Kaner, R. B. - Adv. Mater., 17, p.77 (2005). http://dx.doi. org/10.1002/adma.200400133

17. Kotov, N. A. - Nature, 442, p.254 (2006). PMid:16855576. http:// dx.doi.org/10.1038/442254a

18. Wei, T.; Fan, Z.; Luo, G.; Wang, S. \& Song, L. - Mater. Res. Bull., 44, p.977 (2009). http://dx.doi.org/10.1016/j.materresbull.2008.11.010

19. Lingaiah, S.; Shivakumar, K. N. \& Sadler, R. L. - "Electrospinning of Nylon-66 Polymer Nanofabrics", in: Proceedings of 49th AIAA/ ASME/ASCE/AHS/ASC Structures, Structural Dynamics and Materials Conference, p.1, Schaumburg - USA, abr. (2008).

20. De Ávila Júnior, J. - "Nanomembranas Interlaminares Para Compósitos de Alto Desempenho”, Dissertação de Mestrado, Universidade Federal de Minas Gerais, Brasil (2010).

21. Huang, C.; Chen, S.; Lai, C.; Reneker, D. H.; Qiu, H.; Ye, Y. \& Hou, H. - Nanotechnology, 17, p.1558 (2006). http://dx.doi. org/10.1088/0957-4484/17/6/004

22. Heikkilä, P.; Harlin, A. - Polym. J., 44, p.3067 (2008).

23. Park, S.-W.; Bae, H.-S.; Xing, Z.-C.; Kwon, O. H.; Huh, M.-W. \& Kang, I.-K. - J. Appl. Polym. Sci., 112, p.2320 (2009). http://dx.doi. org/10.1002/app.29520

24. Abramoff, M. D.; Magalhaes, P. J. \& Ram, S. J. - Biophotonics International, 11, p.36 (2004).

25. He, J.-H.; Wan, Y.-Q.; Yu, J.-Y. - Fibers Polym., 9, p.140 (2008). http:// dx.doi.org/10.1007/s12221-008-0023-3

26. Guerrini, L. M.; Branciforti, M. C.; Canova, T.; Bretas, R. E. S. - Mat. Res., 12, p. 181 (2009). http://dx.doi.org/10.1590/S151614392009000200012

27. Prevéy, P.S. - J. Thermal Spray Tech., 9, p.369 (2000). http://dx.doi. org/10.1361/105996300770349827

28. Li, Q.; Jia, Z.; Yang, Y.; Wang, L. \& Guan, Z. - "Preparation and properties of poly(vinyl alcohol) nanofibers by electrospinning”, in: Proceedings of IEEE International Conference on Solid Dielectrics, p.215, Winchester - UK, jul. (2007). http://dx.doi.org/10.1109/ ICSD.2007.4290790

29. Tao, J. \& Shivkumar, S. - Mat. Lett., 61, p.2325 (2007). http://dx.doi. org/10.1016/j.matlet.2006.09.004

30. Wang, C.; Ko, F. K. \& Alcock, M. - "Nanoclay reinforced PLA nanocomposite by electrospinning”, in: Proceedings of 24th Annual ASC Technical Conference, p.1, Newark - USA, set. (2009).

Enviado: $27 / 08 / 11$

Reenviado: 11/03/12 Aceito: 18/03/12 